\title{
Non-White donors and kidney transplant outcomes: a population-cohort study of transplant registry data
}

\author{
Bhavini Pisavadia ${ }^{1}$, Adam Arshad², Imogen Chappelow², Peter Nightingale ${ }^{3}$, Benjamin \\ Anderson ${ }^{1}$, Jay Nath ${ }^{4}$, Adnan Sharif ${ }^{2,4}$
}

1Department of Nephrology and Transplantation, Queen Elizabeth Hospital, Edgbaston, Birmingham, UK, 2University of Birmingham, Birmingham UK, ${ }^{3}$ Department of Medical Statistics, Queen Elizabeth Hospital, Edgbaston, Birmingham, UK, ${ }^{4}$ University of Birmingham, Birmingham, UK.

\section{Abstract}

Kidneys from non-white donors have inferior outcomes, but it is unclear if ethnically matched transplants attenuate this observation. In this population-cohort study, we analysed all kidney-alone transplants performed in the United Kingdom between 2003 and 2015. Focusing on the three main ethnic groups (white, black and south Asian) left a cohort of 27,970 for analysis. Deceased-donor kidney transplants comprised $64.5 \%$ of the cohort. Black and south Asian donors comprised bigger proportions as living-donors (3.8\% versus $6.9 \%$ respectively) compared to deceased-donors $(1.1 \%$ and $1.7 \%$ respectively). In unadjusted analyses, matched ethnicities between donors and recipients resulted in better outcomes for delayed graft function, one-year creatinine, graft and patient survival but these differed by ethnicity matches. Compared to white-to-white transplants, death-censored graft survival was worse in black-to-black (HR 1.48, 95\%CI 1.16-1.89, $\mathrm{p}=0.002)$ and similar among Asian-to-Asian (HR 0.95, 95\%CI 0.76-1.18, $\mathrm{p}=0.620)$ transplants but patient survival was better for both black-to-black (HR 0.67, 95\%CI 0.44-1.01, $\mathrm{p}=0.057)$ and Asian-to-Asian (HR 0.65, 95\% CI 0.48-0.87, $\mathrm{p}=0.004)$ transplants. In Cox regression models, compared to white donors, we observed worse graft survival with both south Asian (HR 1.38, 95\%CI 1.12-1.70, $\mathrm{p}=0.003$ ) and black (HR 1.66, 95\%CI 1.30-2.11, $\mathrm{p}<0.001$ ) donated kidneys independent of recipient ethnicity. We observed no difference in patient survival with south Asian donated kidneys but worse patient survival with black donated kidneys (HR 1.68, 95\%CI 1.21-2.35, $\mathrm{p}=0.002$ ). Matching ethnicities was not significant in any Cox regression model. Our data suggests non-white donors have inferior post-transplant outcomes regardless of recipient ethnicity, with no attenuation for ethnically matched transplants.

\section{Introduction}

- Minority ethnics make up $10.8 \%$ of the general population of the United Kingdom, but $22.4 \%$ of total kidney transplants performed and only $5 \%$ of donors on the Organ Donor Register (1).

- While every effort is made to encourage organ donation from minority ethnic communities, kidneys from non-White donors have been shown to have inferior outcomes post transplant.

- However, limitations with these published studies include:

- Studies are non-UK based.

- Effect of donor versus recipient ethnicity has not been explored (e.g. matched ethnicities has been shown to have superior outcomes after heart (2), lung (3) and liver (4) transplantation).

Aim

- To explore outcomes for kidney transplant recipients stratified by donor versus recipient ethnicity to test the hypothesis that non-White donors achieve poorer outcomes for kidney transplant patients regardless of recipient ethnicity.

\section{Methods}

- We retrospectively analysed UK Transplant Registry data for all kidney-alone allograft recipients between April 1st 2003 and March 31 st 2015 (data censored at $1^{\text {st }}$ October 2016).

- We analysed a study cohort of 27,970 where donor or recipient ethnicity was white, black or south Asian.

- Survival analysis was performed with Cox proportional hazards models to estimate risk for kidney allograft loss or death.

\section{Results}

- After exclusion, a total of 27,970 kidney transplant recipients were available for analysis $(23,215,1,679$ and 3,076 kidney transplant recipients of white, black and south Asian ethnicity respectively.

- Median follow up time from kidney transplantation was 1,676 days (IQR 716 to 2,869 days).

- Figure 1 shows unadjusted Kaplan-Meier graft survival plots:

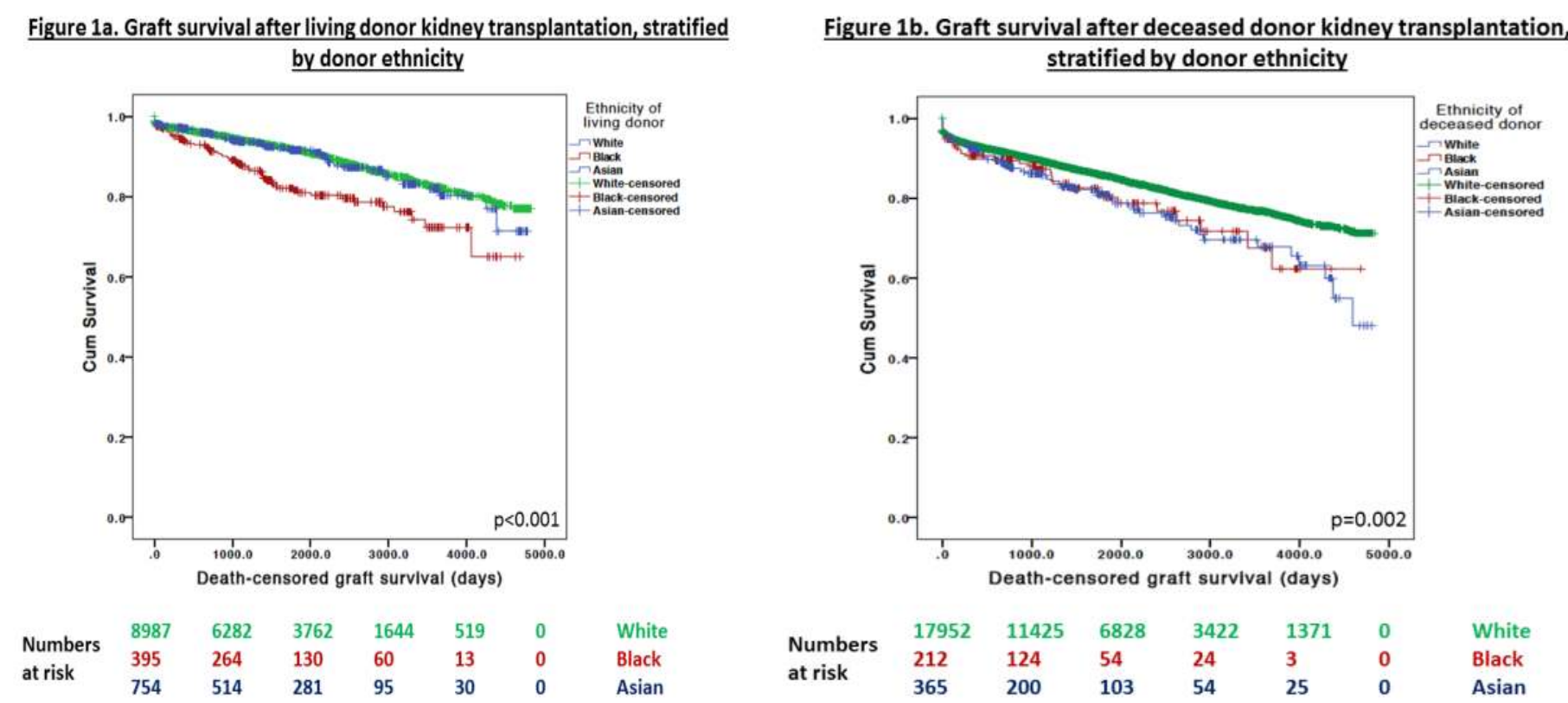

- Figure 1 shows unadjusted graft survival after (a) living or (b) deceased donor kidney stratified by donor ethnicity.

- The following tables show risk for graft loss or death in unadjusted and adjusted Cox regression models (adjusted for donor, recipient and transplantrelated variables):

Table 1. Unadjusted analyses of risk for graft loss with ethnicity matches

\begin{tabular}{|c|c|c|c|c|c|}
\hline \multirow{2}{*}{$\begin{array}{c}\text { Donor to Recipient } \\
\text { Ethnicity }\end{array}$} & \multirow{2}{*}{$\mathbf{n}$} & \multicolumn{2}{|c|}{ Graft survival } & \multicolumn{2}{|c|}{ Patient survival } \\
\hline & & $\mathrm{HR}(95 \% \mathrm{Cl})$ & $\mathrm{P}$ value & $\mathrm{HR}(95 \% \mathrm{Cl})$ & $P$ value \\
\hline Asian to Asian & 734 & $0.95(0.76-1.18)$ & 0.62 & $0.65(0.48-0.87)$ & 0.004 \\
\hline Asian to Black & 50 & $1.86(1.03-3.36)$ & 0.040 & $0.43(0.11-1.73)$ & 0.24 \\
\hline Asian to White & 196 & $1.42(1.00-2.03)$ & 0.051 & $0.88(0.53-1.46)$ & 0.61 \\
\hline Black to Asian & 62 & $1.46(0.76-2.81)$ & 0.26 & $2.04(1.02-4.09)$ & 0.044 \\
\hline Black to Black & 387 & 1.48 (1.16-1.89) & 0.002 & 0.67 (0.44-I.0I) & 0.057 \\
\hline Black to White & 132 & $1.33(0.86-2.06)$ & 0.21 & $1.08(0.61-1.90)$ & 0.79 \\
\hline White to Asian & 2280 & $1.26(1.12-1.41)$ & $<0.001$ & $1.22(1.07-1.40)$ & 0.004 \\
\hline White to Black & 1242 & $1.56(1.36-1.79)$ & $<0.001$ & $0.97(0.79-1.18)$ & 0.75 \\
\hline White to White & 22887 & 1.00 & - & 1.00 & - \\
\hline
\end{tabular}

Table 2. Cox proportional hazards model for risk of graft loss and death*

\begin{tabular}{|c|c|c|c|c|}
\hline \multicolumn{2}{|c|}{ Parameter } & Risk for graft loss & $95 \% \mathrm{CI}$ & $P$ value \\
\hline \multirow{3}{*}{ Donor ethnicity } & White & 1.00 (reference) & - & - \\
\hline & Black & 1.66 & $1.30-2.11$ & $<0.001$ \\
\hline & South Asian & 1.38 & I.I2-I.70 & 0.003 \\
\hline \multicolumn{2}{|c|}{ Parameter } & Risk for death (HR) & $95 \% \mathrm{Cl}$ & P value \\
\hline \multirow{3}{*}{ Donor ethnicity } & White & 1.00 (reference) & - & - \\
\hline & Black & 1.68 & $1.21-2.35$ & 0.002 \\
\hline & South Asian & 0.88 & $0.64-\mid .21$ & 0.423 \\
\hline
\end{tabular}

*Adjusted for donor age, donor smoker, donor sex, donor ethnicity, recipient age, recipient sex, recipient ethnicity, recipient diabetes, waiting time, cold ischemic time, sensitization (calculated reaction frequency), graft number, HLA mismatch, delayed graft function, rejection, type of donor and ethnicity matching between donors/recipients.

\section{Discussion}

- Our findings are consistent with existing literature showing inferior outcomes associated with kidneys from non-White donors, regardless of deceased or living kidney donation

- We did not identify any survival benefits following donor-recipient ethnicity matched transplants as shown in non-renal transplant literature.

- However, kidney transplantation from any donor ethnicity is likely to still have superior survival outcomes compared to dialysis for patients with kidney failure and patients should be appropriately counselled as such.

\section{References}

1. http://www.odt.nhs.uk/pdf/activity-report/bame organ donation and transplantation.pd 2. Kanter et al. Donor-Recipient Race Mismatch and Graft Survival After Pediatric Heart Transplantation. Ann Thorac Surg 2009; 87: 204-210

Allen et al. Impact of Donor-Recipient Race Matching on Survival After Lung Transplantation: Analysis of Over 11,000 Patients. J Heart Lung Transplant 2009; 28: 1063-1071

4. Pang et al. The effect of donor race on the survival of Black Americans undergoing liver transplantation for chronic hepatitis C. Liver Transpl 2009; 15: 1126-32

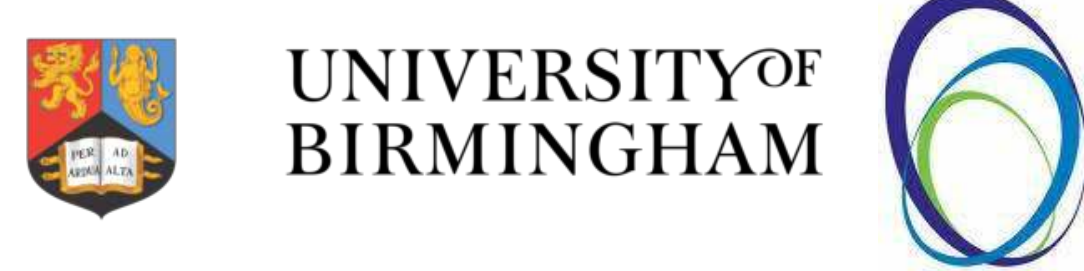

Queen Elizabeth Hospital Birmingham 